\title{
EL ANÁLISIS DE CONTENIDO Y EL DESARROLLO DEL GUSTO MUSICAL: EL CASO DE LAS REVISTAS MUSICALES

\author{
Amparo Porta Navarro
}

\author{
Universidad Jaume I \\ porta@edu.uji.es
}

\begin{abstract}
Este artículo muestra algunos aspectos de la investigación realizada sobre los contenidos de revistas musicales, siendo su objetivo conocer qué es música para ellas y con que se relaciona. La metodología, cualitativa, utiliza como modelo el interaccionismo simbólico. Los resultados muestran que las revistas hablan de cantantes, canciones, muy poco de música y mucho de la propia revista, tienen un componente emocional alto, una escasa apuesta por la creación y un nivel crítico bajo. El estudio puede ayudar a dar visibilidad a algunos contextos de la música como fuentes del currículum que permitan orientar la búsqueda de respuestas desde la educación musical a este imaginario de alto impacto.
\end{abstract}

This article presents some aspects of a study of the content of music magazines. It aims at understanding the magazines' approach to music and what they relation with it. The qualitative methodology is based on symbolic interactionism. The results show that these magazines talk about singers and songs, little about music, and very much about the magazine itself. They evidence a strong emotional charge, limited interest in individual creation, and little criticism. The study can make some music contexts more visible as curriculum sources, to help finding answers from music education into these high-impact imaginary.

\section{INTRODUCCIÓN}

\section{Planteamiento del problema}

Este trabajo tiene por objeto dar a conocer algunos resultados de la investigación realizada en 2007 sobre los contenidos de revistas musicales de gran tirada dirigidas a la población más joven. Nuestra intención ha sido hacer visible el imaginario musical de estas revistas como medio cultural, así como transmisor de ideas y gustos de la franja de población que más cantidad de música ha escuchado en la historia, y también, la más mirada por industrias y productores por ser exclusivamente consumidora. Conocer de qué hablan las revistas musicales es la finalidad de este artículo porque desde la investigación en educación musical compartimos su público, ya que sus clientes potenciales son también el sujeto de la educación.

Ninguna época ha sido tan sensible al fenómeno música como esta, sin embargo, los vaivenes a los que los diseños curriculares europeos y españoles la tienen sometida, indican un punto de fricción y desajuste entre la música que se escucha y el valor educativo que se atribuye a la misma. Este artículo trata de conocer los contenidos de algunos espacios masivos de la difusión musical, porque son conformadores de aspectos relevantes para la educación musical como el gusto y sus valores asociados, la identificación entre grupos de iguales y otros muchos que utilizan la música como protagonista o reclamo. Y también, porque por medio de ellas, se construye parte del imaginario de la población joven, por lo que dicen, por lo que callan, desde dónde lo dicen y a quiénes lo dicen (PORTA, 2007). No es una banalidad querer conocer los espacios comunicativos de una de las grandes industrias de los siglos XX y XXI, que miden por millones sus lectores y también sus ganancias, utilizan grandes formatos, selecciones aparentemente cuidadosas de sus contenidos impresos en papel couché, y altas dosis de publicidad manifiesta y encubierta. Las revistas musicales tienen la mirada puesta en sus clientes, constituyendo un macro contexto industrial y mediático que convive en clara desigualdad comunicativa con la música que llega a través de los niveles obligatorios de la escolaridad. La educación musical tiene una valoración institucional constante e inexorablemente disminuida, que comenzó siendo alta con la aparición de la LOGSE y que hoy, veinte años y tres leyes después, ocupa su lugar más bajo. La actual ley de educación habla de la vertiente comunicativa de la música de forma explícita (BOE, 2006 y 2007), y también lo hace aunque no la nombre, cuando se pronuncia sobre la expresión de lenguajes contemporáneos y universales así como cuando habla de la educación como correa de transmisión cultural, patrimonial y de valores (GIMENO SACRISTÁN, 1983). 
Desde la animación sociocultural diferentes trabajos se han aproximado a la búsqueda de recursos metodológicos, como el presentado por J. E. PÉREZ; J. EsCUDERO (2004) con el fin de posibilitar proyectos sociales más adecuados a las necesidades de las comunidades particulares. Sobre las preferencias musicales juveniles se han interesado diferentes trabajos en el ámbito internacional, como LITLE y ZUCKERMAN (1986), en cuya obra se manifiestan de forma rotunda sobre la preferencia de los jóvenes por los géneros y estilos populares del siglo XX. Entre los trabajos sobre la audiencia y la creación del gusto es de destacar el texto de TANNER (2008) sobre las preferencias musicales de los adolescentes realizada en un centro de Educación Secundaria de Toronto. El autor investiga el fenómeno desde la estratificación cultural y subcultivos adolescentes, manifestando la necesidad de explorar conexiones entre los gustos musicales y la actividad de los grupos de iguales, estableciendo relaciones entre las experiencias en la escuela y el capital cultural de partida. Igualmente, HeNNION (2010) dice que el gusto es un comportamiento. Reproducir, escuchar, grabar, hacer que otros escuchen música... vienen a ser algo más que la realización de un gusto que ya existía. Por ello, dice, la vinculación entre los aficionados y la forma de hacer las cosas se combinan y forman subjetividades creando historias que sobrepasan a las propias obras. Entendido de esta manera, el gusto del aficionado ya no se considera una elección arbitraria explicada por razones sociales ocultas, más bien, es una acción colectiva, cuyo análisis ayuda a entender la manera en la que nos hacemos sensibles a las cosas, a nosotros mismos, a las situaciones y a los momentos. Finalmente, desde el campo de la etnomusicología la aportación de PELINSKY (2007) explora relaciones teóricas entre ecopsicología y fenomenología, en vistas a su aplicación a la percepción del entorno sonoro. El autor establece para ello una correlación entre el objeto de la experiencia y sus maneras de darse a la conciencia, sus posibles aplicaciones a la percepción sonora así como sus formas de aproximación. Para ello estudia las relaciones del organismo con el entorno y la escucha musical, buscando un acercamiento entre las relaciones entre percepción y acción. En el ámbito nacional, HeRrera (2010) muestra en su trabajo los estilos musicales más consumidos por los adolescentes, siendo el más escuchado el pop, seguido del reggaeton, el hip-hop y el rap, y señalando un dato de alto interés en el tema que nos ocupa: la educación formal tiene sus preferencias en los estilos musicales menos consumidos. De igual modo, sobre la influencia de los grandes medios de difusión, las investigaciones realizadas por nuestro propio grupo sobre la televisión han mostrado las características de la música que escuchan los niños en la televisión en una muestra latinoamericana, concluyendo en su tendencia reduccionista (PORTA, 2010). Finalmente, desde la vertiente formativa de la educación, la revisión de la bibliografía muestra en España escasos estudios, centrando sus trabajos en soportes, aspectos tecnológicos y de recursos en el aula en los números 23 de Comunicar y 12 de Eufonía. Por todo ello podemos concluir diciendo que no se dispone de indicadores empíricos que aseguren un buen conocimiento del campo de estudio.

\section{El estado de la cuestión}

Nuestro objeto de estudio es la imagen de música con la que se identifican los grandes medios masivos de difusión dirigidos a la infancia y la juventud. La música es un fenómeno del que todo el mundo participa y habla, pero del que existen escasos trabajos que la expliquen desde la educación. Sin embargo, como indicador de influencia y modelador social está vinculado a la especificidad de su propio lenguaje como producción de sentido en el espectador (PORTA, 2007). Por ello se hace necesario conocer cuáles son sus contenidos musicales, porque producen sentido y determinan una parte importante de la construcción social del mundo en la infancia (VYGOSTKI, 1981). Sin embargo, la construcción del imaginario utiliza en nuestra época un vehículo mediático e industrial de difusión musical que requiere de un estudio que tenga en cuenta sus peculiaridades e influencia. 
FRUTOS ESTEBAN (2008) considera que el desarrollo de técnicas de análisis sistemático de mensajes mediáticos es uno de los desafíos metodológicos en las próximas décadas. El análisis de contenido, dice, como método que permite investigar con detalle y en profundidad cualquier material de la comunicación humana puede ser una herramienta esencial en la descripción ordenada de repertorios comunicativos y culturales. Y concluye el autor diciendo que puede ser un método de arranque en la constitución de una ecología cultural basada en la investigación de la comunicación social.

Sobre el imaginario, un concepto propuesto inicialmente por CASTORIADIS (1989), ha sido vinculado a la filosofía marxista y ampliamente extendido en las ciencias sociales. Este concepto es tomado en nuestro texto como sinónimo de cosmovisión construida por la propia sociedad y convertido en una parte importante de la inmersión cultural del niño y el adolescente actual. Desde nuestra temática, diferentes autores y corrientes se han interesado por este concepto. Así, LACAN (2009) se posiciona desde el psicoanálisis sobre las construcciones simbólicas diciendo que un proceso de pensamiento del orden simbólico involucra siempre, una base o soporte en lo real y una representación en el registro de lo imaginario. Igualmente, Del Río y DEL Río (2008) dicen, hablando de las nuevas formas de alfabetización, que hay que considerar la alfabetización del imaginario como una de las formas necesarias en la educación actual. El problema de la calidad de los contenidos, explican, hablando de la televisión y su impacto en la infancia, está emergiendo en los últimos años como uno de los factores centrales para comprender los lastres o los déficits más preocupantes que afectan al empeño educativo. Los autores toman como referencia los contenidos televisivos y habla de que la dieta cultural está constituida por el conjunto de elementos culturales disponibles para un individuo, grupo o cohorte generacional a lo largo de su desarrollo (ontogénesis). Del Río y Del Río (2008) terminan diciendo que esta dieta recibida está a su vez determinada por la dieta ofertada, que constituye el total de productos culturales producidos, distribuidos o puestos en el espacio cultural.

El carácter altamente mediático, industrial y de consumo de la música hoy en día requiere, de forma obligada, conocer algo más que el gusto de los niños y los adolescentes, requiere también conocer los factores responsables de su construcción, cuales son los elementos que maneja y con qué se relacionan. Este gusto musical resultante se crea con los elementos sonoros y musicales que la sociedad le proporciona y no otros, y con todo ello construye el imaginario. Esta cosmovisión del mundo es generada por el hábitat sonoro más próximo y también por los grandes medios de comunicación. El primero lo constituyen la lengua materna en música y está formado por canciones, sonidos, instrumentos, melodías y armonías del propio entorno más próximo, escuchado de persona a persona en su propio contexto natural y afectivo; mientras el segundo lo hace por criterios industriales basados en la edición y la producción, que determinan y seleccionan una gran parte de la música que escucha, y a su vez fagocitan una parte del hábitat aumentando la globalización y disminuyendo la diversidad como valor (PORTA, 2011).

Por ello decimos que las revistas musicales representan la música actual más consumida, constituyéndose en referente de valores, y construyendo una parte importante del gusto, siendo un importante referente para millones de niños y jóvenes. Por último, suponen en sí mismas, un excelente resumen del gran aparato mediático de la música pop de las tres últimas décadas, compuesta por canales de televisión y radio, relaciones con discográficas, multinacionales y los grandes circuitos de conciertos.

\section{La oferta musical}

Nuestra intención es disponer de un primer mapa que nos permita conocer los contenidos de los que hablan algunas revistas musicales, porque con ellas se crea una representación del mundo al que siempre sustituyen como una opción entre otras posibles, y también porque a través de sus 
referencias, elecciones y omisiones dan forma al universo de la música que recibe el niño y joven espectador.

Nuestra pregunta de investigación es: ¿Cuáles son los contenidos musicales de las revistas musicales y con qué se relacionan? Esta pregunta se vincula directamente con la educación musical ya que en el imaginario quedan alojados o excluidos aspectos y contenidos educativos de primer orden, como la expresión musical, la producción sonora, la comprensión cognitiva de la música así como su sentido diacrónico y de conexión cultural en la sociedad de hoy en día. Se hace necesario un buen diagnóstico sobre las características del nuevo hábitat sonoro surgido de la postmodernidad para establecer un equibrio entre las fuerzas sociales y la educación. Por ello, los diseños curriculares han de hacerse sensibles a factores culturales e industriales con repercusiones indiscutibles en la toma de decisiones de la teoría y práctica educativa.

Si las revistas mueven una importante industria con tiradas millonarias multinacionales y reúnen bajo su paraguas a jóvenes y adolescentes de todo el mundo, sus contenidos interpelan a la educación. El currículum siempre es un supuesto del hábitat sonoro, y desde la teoría y práctica educativa, muchas veces, tenemos la tentación de querer decidir por los demás qué es música para cada uno, pero, finalmente, ese carácter de escucha significativa vendrá dado por la lectura que de ello haga su verdadero protagonista: el sujeto de la educación.

\section{Del universo de referencia a la selección de categorías}

La toma de decisiones para la selección de indicadores es uno de los retos de este trabajo. Hemos seguido un proceso inductivo porque queremos beber directamente en las fuentes de la música que niños y jóvenes escuchan. Por todo ello hemos tomado cinco decisiones determinantes para llegar a los indicadores finales del estudio, son las siguientes (Fig. 1):

- Toma de decisión 1. Entorno pop/rock. Hemos elegido como universo la música más escuchada por niños y jóvenes, la música popular contemporánea, por su capacidad identificatoria y proyectiva, porque establece lazos afectivos y comunicativos entre los grupos de iguales, por su difusión masiva y por sus efectos de refuerzo.

- Toma de decisión 2. Revistas musicales. La elección de las revistas musicales ha sido motivada porque suponen un resumen de las tendencias musicales más consumidas hoy en día y porque son el objeto impreso resultante de las programaciones de cadenas de televisión y radio, giras de conciertos y relaciones discográficas con presencia en todo el mundo.

- Toma de decisión 3. Los editoriales. La tercera toma de decisiones ha provenido de la elección de los textos de la muestra. Los editoriales firmados por los directores de las revistas musicales más importantes suponen un resumen de cada uno de los números con indicación de los apartados y contenidos destacados, pero no sólo eso, describen la línea editorial de sus valores, aspiraciones y opciones tanto culturales, como sociales y musicales. Mediante los editoriales podemos acceder a las aspiraciones de las propias revistas, sus expectativas y efectos de refuerzo, y también a todos los elementos que por callados, desaparecen de su universo de valores. Los editoriales describen mucho más que la oferta y sus elementos destacados, describen aquellos contenidos que tienen cabida como posibles dentro de la revista y cuales, por no apoyados, no tienen presencia en ellas, ni en las cadenas televisivas y radiofónicas a las que representan, ni en las giras a las que dan cobertura con su patrocinio y publicidad.

Toma de decisión 4 y 5. Análisis de contenido y categorías. Las dos últimas decisiones tienen carácter metodológico y están basadas en el interaccionismo simbólico. La cuarta fue la elección del análisis de contenido con ayuda del programa Atlas.ti, para poder acceder de forma inductiva a sus contenidos y no a su previsión o suposición realizada sobre un análisis previo, todavía no realizado 
(GLASER y STRAUSS, 1967). Ambas por su carácter metodológico serán explicadas en el apartado correspondiente.

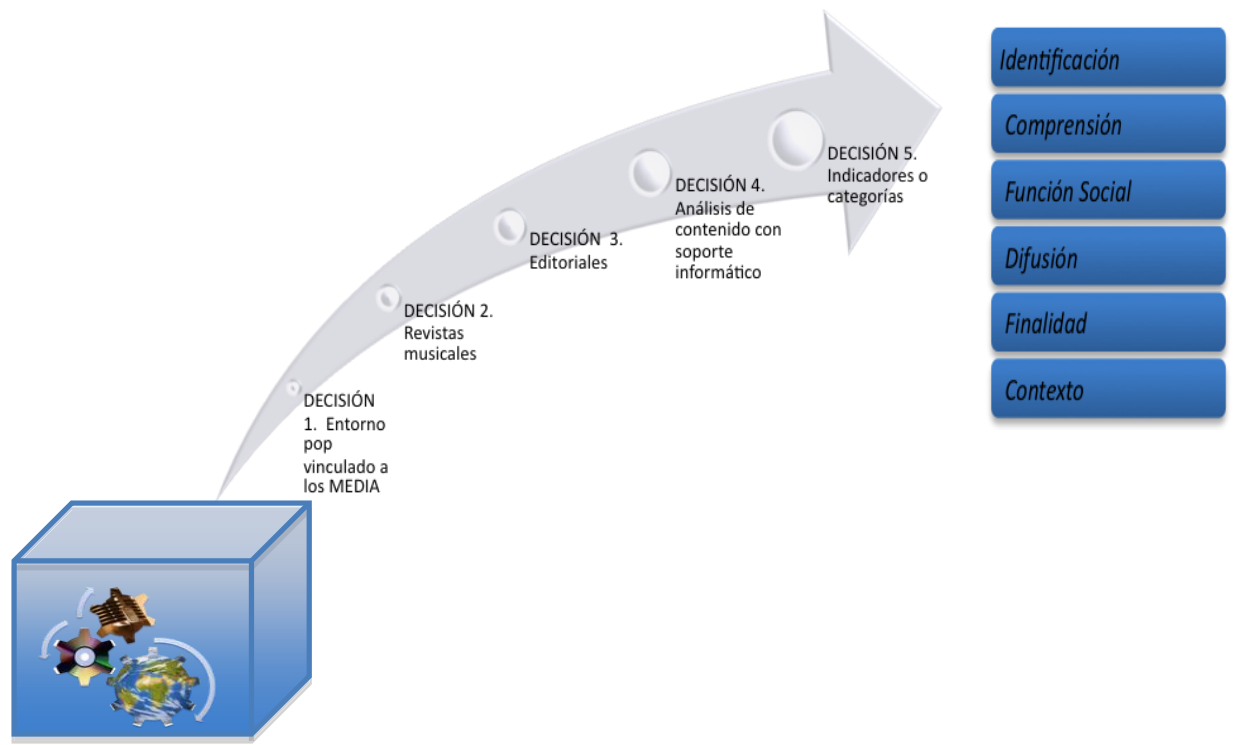

Fig. 1: Del universo de referencia a la selección de indicadores

\section{APROXIMACIÓN METODOLÓGICA: ANTECEDENTES}

Nuestro tema se interesa por la música desde el lugar del sujeto en la interacción, por lo tanto depende, como construcción social, de las ciencias sociales y necesita establecer un punto de vista o modelo de análisis para su comprensión que en nuestro caso será cualitativo. El fenómeno músical hoy en día es omnipresente en nuestra sociedad, por ello es necesario hacer una selección puesto que, tal y como afirma SCHÜTZ (1967), los hechos sólo se hacen relevantes por medio de su selección e interpretación. Para GoOdman (1978), el mundo se construye socialmente mediante formas diferentes de conocimiento, desde el cotidiano hasta la ciencia y el arte como formas de crear el mundo diferentes. Según SCHUTZ (1962; 59), "los constructos de las ciencias sociales son constructos de segundo grado, es decir, constructos de los constructos, hechos por los actores en la escena social". En este sentido, el autor mantiene que "la exploración de los principios generales con arreglo a los cuales el hombre organiza sus experiencias en la vida diaria, y especialmente las del mundo social, es la primera tarea de los métodos de las ciencias sociales" (SCHUTZ, 1962:59). En particular, la investigación de la ciencia social se enfrenta al problema de que encuentra el mundo que quiere estudiar siempre y sólo en las versiones de este mundo existentes en el campo o construidas por sujetos interactuantes. Así pues, no es suficiente la elección de la investigación cualitativa, necesitamos de un modelo para orientar nuestro trabajo y comprender su significatividad en la realidad actual, por todo ello, nuestra siguiente tarea será conocer cuáles son las referencias teóricas o paradigmas para hacer una lectura apropiada del fenómeno.

\section{Elección del paradigma}

Nuestra opción será el interaccionismo simbólico porque estudia la interacción como espacio de discusión y construcción del conocimiento, considerando este paradigma que las personas son responsables de los cambios sociales y lecturas del mundo (GARFINKEL, 1967). Se sustenta fundamentalmente en una filosofía pragmática y explora las comprensiones de la cultura como la matriz significativa que guía nuestras vidas (CROTTY, 1998). Fue desarrollado desde la tradición filosófica del pragmatismo americano, representada en figuras como DEWEY (2008) y JAMES 
(1961), y se sitúa en el ángulo de los sujetos que estudia CROTTY (1998). Desde su perspectiva, todas las organizaciones, culturas y grupos están constituidos por actores envueltos en un proceso constante de interpretación del mundo que les rodea. Aunque estas personas pueden actuar dentro del marco de una organización, cultura o grupo, son sus interpretaciones y definiciones de la situación las que determinan la acción, y no normas, valores, roles o metas (TAYLOR y BOGDAN, 1992:25). BLUMER (1969) afirma que el interaccionismo simbólico reposa sobre tres premisas básicas:

- Las personas actúan respecto de las cosas e incluso respecto de las otras personas, sobre la base de los significados que estas cosas tienen para ellas.

- Los significados son productos sociales que surgen durante la interacción.

- Los actores sociales asignan significados a situaciones, a otras personas, a las cosas y a sí mismos a través de un proceso de "interpretación".

Este proceso tiene dos pasos distintos. Primero, el actor se indica a sí mismo las cosas respecto de las cuales está actuando, en nuestro caso la música. En segundo lugar, en virtud de este proceso de comunicación consigo mismo, la interpretación se convierte en una cuestión de manipular significados (BLUMER, 1969:5). Esta corriente está interesada en preguntas como las siguientes (TAYLOR y BOGDAN, 1992):

- ¿Cómo se definen las personas a sí mismas y a otros, a sus escenarios y actividades?

- ¿Cómo se desarrollan y cambian las definiciones y perspectivas de la gente?

- ¿Cuál es el ajuste entre las diferentes perspectivas de distintas personas?

- ¿Cuál es el ajuste entre las perspectivas de la gente y sus actividades?

- ¿Cómo tratan las personas con las discrepancias entre sus perspectivas y actividades?

Estas inquietudes por las que se interesa el interaccionismo simbólico definen el primer nivel de concreción de nuestro estudio, aproximándonos a los indicadores o categorías de los contenidos de las revistas musicales de mayor tirada. Éstos son los que nosotros proponemos como categorías a las preguntas planteadas por los autores para encontrar indicadores de sus contenidos:

\begin{tabular}{|l|l|}
\hline Preguntas planteadas & Indicadores \\
\hline ¿Cómo se definen las personas a sí mismas y a otros, a sus escenarios y sus actividades? & Identificación \\
\hline ¿Cómo se desarrollan y cambian las definiciones y perspectivas de la gente? & Referencia \\
\hline ¿Cuál es el ajuste entre las diferentes perspectivas de distintas personas? & Posición crítica \\
\hline ¿Cuál es el ajuste entre las perspectivas de la gente y sus actividades? & A qué mundos remite \\
\hline ¿Cómo tratan las personas con las discrepancias entre sus perspectivas y actividades? & Posición crítica \\
\hline
\end{tabular}

\section{Tabla 1: Primer nivel de concreción}

Así, las relaciones musicales del niño y el adolescente con el entorno cotidiano como universo referencial del gusto tienen una aproximación a través del análisis de contenido de las revistas musicales, concretado a través de cinco grandes indicadores o categorías de análisis: identificación, comprensión, función social, difusión, finalidad y contexto.

El siguiente paso ha sido la elección metodológica que obtuvimos por medio del análisis cualitativo (ÁlvAREZ, 2003; BÁEZ, 2007; FLICK. 2007; PATTON, 2002). Esta aproximación tiene por objeto el conocimiento de la realidad a la que accede a través del discurso, así como la comprensión de las interacciones que se dan en la realidad cultural y los mecanismos que intervienen. Estos planteamientos tienen una serie de elementos comunes entre sí. En primer lugar son propuestas que abordan al ser humano y los mecanismos por los cuales configura su existencia. En segundo lugar, diferentes autores coinciden en que tratan las cuestiones relativas a las formas en que la persona interactúa con su entorno y abordan sus relaciones con los otros. Por último comparten una cierta unidad de criterio en cuanto a que hay realidades más o menos objetivas, pero que son las 
experiencias personales y los mecanismos de percepción de las realidades y su incorporación a la conciencia los que configuran las realidades.

\section{Preguntas de investigación y objetivo del estudio}

La pregunta que centra nuestra investigación se refiere a cuáles son los contenidos musicales de las revistas musicales y con qué se relacionan. Para contestar dicha pregunta, y siguiendo el procedimiento inductivo propuesto por TAYLOR y BOGDAN (1992), hemos establecido un diálogo entre lo que sabemos, lo que leemos en las revistas y lo que buscamos. Para contestar nuestra pregunta de investigación necesitamos saber:

- De qué música hablan las revistas, qué dicen y cuáles son sus temas.

- Cuál es el recorrido de la música desde la creación hasta su difusión.

- Para qué lo hacen y dónde lo muestran.

- Cuándo y dónde ocurren los hechos y acontecimientos musicales.

- Qué actividades giran a su alrededor.

- Qué les preocupa.

- A qué mundos remite.

Surgiendo de estas preguntas las seis categorías resultantes: identificación, comprensión, función social, difusión, finalidad y contexto.

\section{METODOLOGÍA}

\section{Elección del material y la metodología}

La elección de las revistas Rolling Stone y La Revista 40 está motivada porque son un testigo de excepción de gustos y tendencias actuales sobre música popular no especializada, tener una difusión masiva y estar vinculadas a los grandes medios de comunicación de masas. El carácter multinacional de la revista Rolling Stone, con 30 ediciones en todo el mundo y más de quince millones de lectores la sitúan en primera línea de la influencia social de la música. Por otro lado, $L a$ Revista 40 sintetiza en España todo un holding mediático musical compuesto por conciertos, emisoras de radio, programas de televisión, programadores de éxitos secuenciados, sectores publicitarios de alta influencia, presencia de discográficas, giras promocionales, canalización de nuevos soportes, apoyos a compañías discográficas y relación con los medios afines. Hacer visible el espacio en el que se difunde la música de forma masiva es el objeto de este texto. Por ello hemos optado por uno de los grandes espacios donde se muestra hablando de sí misma y de todo aquello con lo que se le relaciona: las revistas musicales. A esta primera elección han seguido otras como la elección de la muestra (los editoriales) y el análisis de su contenido con soporte informático.

\section{Descripción del procedimiento}

Como pasa con cualquier forma de análisis cualitativo, no existe un método único de análisis del contenido, ya que este varía según el tipo de texto analizado y los objetivos con los que se realiza (BÁEZ, 2007). El procedimiento de análisis seguido por nosotros ha consistido en la preparación del material seleccionando: todos los editoriales firmados por los directores de las revistas Rolling Stone y La Revista 40 publicados en 2007. Una vez seleccionado, el primer paso ha sido la lectura, después hemos procedido a su codificación y, finalmente, a su selección en unidades de análisis o categorías. Esta fase, tal y como coinciden todos los analistas cualitativos, es la más importante puesto que constituirán la estructura de nuestro informe o conclusiones (KELLE, 1995). Para la 
validez de los resultados se han tenido en cuenta la "exhaustividad", porque se han considerado todos los elementos, es "representativa" dado que el discurso está "saturado" y ha sido "clausurado", todas sus aportaciones son "homogéneas" y es "pertinente" al objetivo que pretendíamos en un principio.

\section{El programa Atlas.ti}

Atlas.ti, de MuHR, profesor de la Universidad de Berlín, es un programa informático para la investigación cualitativa (MUÑOZ, 2003). Se basa en la teoría fundamentada (GLASER. y STRAUSS, 1967) y su proceso implica tres etapas: 1) la categorización de la información, 2) la estructuración o creación de una serie de redes de relaciones entre categorías que representan gráficamente posibles estructuras, 3) la teorización propiamente dicha. La codificación asigna dos números: el primero muestra el número de citas codificadas con el mismo código ("relevancia de la cita"); y el segundo, el número de otros códigos y categorías conectadas a la estructura ("densidad teorética"). En el estudio hemos utilizado como documentos primarios los editoriales de las revistas Rolling Stone y La Revista 40 del año 2007. A partir de ello hemos seleccionado las citas que hablan de música, en un total de 238 que hemos codificado en 86 códigos agrupados finalmente en seis categorías. Con todo ello hemos constituido 44 redes de las que mostramos aquí una pequeña selección.

\begin{tabular}{|c|c|c|c|}
\hline \multicolumn{2}{|l|}{ Revista } & Rolling Stone & La Revista 40 \\
\hline \multirow{7}{*}{$\begin{array}{l}\text { Datos de la } \\
\text { revista }\end{array}$} & periodicidad & mensual & mensual \\
\hline & editorial & Prisa & Prisa \\
\hline & director & Pedro Javaloyes & Miguel A. Bargueño \\
\hline & temática & musical y estilo & musical \\
\hline & $\mathrm{n}^{\mathrm{o}}$ de páginas & 150 & 150 \\
\hline & muestra & 12 revistas $(2007)$ & 12 revistas $(2007)$ \\
\hline & editoriales & meses $4,5,6,10,11,12$ & meses $3,4,5,6,7,9,10,11,12$ \\
\hline \multicolumn{2}{|c|}{ Titulo de los editoriales } & $\begin{array}{l}\text { "El futuro ya está aquí (y suena)" } \\
\text { "Rolling Stone, una manera" } \\
\text { "El top de los odiados" } \\
\text { "Los piratas del Rock" } \\
\text { "Porqué elegimos a Bruce" } \\
\text { "Cosas que cambió Rolling" }\end{array}$ & $\begin{array}{l}\text { "¿Qué sabemos de Dani Martín?” } \\
\text { "La gira más sorporendente" } \\
\text { "Historia de un paraguas" } \\
\text { "Escuela de rock" } \\
\text { "Humor amarillo" } \\
\text { "Músicos solidarios" } \\
\text { "España "82" }\end{array}$ \\
\hline \multicolumn{2}{|c|}{ Procedimiento } & \multicolumn{2}{|c|}{$\begin{array}{l}\text { Localización del material. } \\
\text { Sección y recopilación de contenido. } \\
\text { Organización, preparación y digitalización de documentos. }\end{array}$} \\
\hline
\end{tabular}

Tabla 2: Estudio del contenido de las revistas. Descripción y muestra

\section{ANÁLISIS DE LOS DATOS: RESULTADOS POR CATEGORÍAS}

\section{¿De qué hablan? Identificación y referencia}

Las revistas toman postura respecto a la música. En sus editoriales no siempre la nombran pero, cuando lo hacen, hablan preferentemente de cantantes, de canciones y de la propia revista que es lo más nombrado.

La apuesta principal de las revistas son ellas mismas. Rolling Stone se nombra 19 veces, 18 de forma manifiesta y 1 de forma latente. La Revista 40 se nombra 25 veces y 3 como Los 40 principales que da nombre a la emisora de radio y televisión, eligiendo el número 40 como refuerzo para hablar de edades, selecciones musicales y otros. En cuanto al contenido, la mayor parte lo 
ocupan los cantantes, que los nombra por medio de la identificación "Lo que son", y también por medio de referencias, "lo que hacen”. Sobre la identificación, en la muestra, se utilizan 26 atributos musicales y 21 no musicales. De estos últimos, 7 son de carácter y 8 de rasgos físicos, de los cuales 6 corresponden a mujeres. En cuanto a las referencias, hablan de conmover, propiciar el sentido crítico y ser precursores. Finalmente, las canciones se identifican por su valoración musical (las mejores), o por estar seleccionadas en un disco (single).

\section{¿Qué dicen? Comprensión}

Las revistas se califican a sí mismas como musicales pero hablan poco de música. En los editoriales examinados aparecen 18 citas que aluden a algunos, muy pocos conceptos musicales: 13 sobre géneros musicales y 5 sobre la forma musical, el estribillo, la escucha y percepción auditiva, así como de la música como forma de expresión que culmina con la producción industrial.

\section{Función social, difusión y finalidad}

Las revistas remiten a un mundo exterior donde ellas dejan de ser el punto de referencia, y toman la defensa del planeta, la sociedad y el grupo de iguales como territorios a defender. Los espacios sociales que muestran son: la liberación de la mujer en La Revista 40, algunos temas políticos y dar voz a una generación, representado por la fotografía de John Lennon y Yoko Ono en Rolling Stone. En cuanto a las actividades profesionales, cuya búsqueda ha sido realizada contestando a la pregunta ¿Qué hacen los otros? Mencionan cuatro profesiones: gestores, intermediarios y servicios nombrados en plural), y creadores nombrados en singular. Sobre la difusión, destacan el último disco y el directo, siendo sus protagonistas los cantantes a los que dedican entrevistas de varias páginas, anuncian en sus portadas y ocupan la entrevista "estrella".

En la difusión, naturalmente, hablan de cantidades. Éstas son las encontradas en orden decreciente:

- 2.000 millones de telespectadores

- Un millón de descargas

- Quince minutos de fama

- Decenas de músicos

- Los únicos de un cartel

¿Para qué lo hacen? Finalidad. La finalidad es el éxito por medio de las listas y la venta, aunque esta última aparece velada en los textos. Por ello hay que observarla como resultado de una fuerza entre otras.

Las referencias al éxito se hacen explícitas sólo una vez, y el fracaso no existe en el mundo real (el de las revistas) sólo fracasan Los Simpson (el de la ficción) tal como podemos ver en los dos únicos textos que hablan de fracaso.

- Los Party Posse inician una fulgurante escalada al estrellato.

- Los Party Posse ven cómo el final de su aventura musical se precipita de un modo ridículo y desternillante.

- Nefasta carrera discográfica. 


\section{El contexto}

Hemos observado cinco contextos: espacial, temporal mediático, grupo de iguales y mundos posibles. Destacamos algunos de ellos por sus implicaciones educativas. "El presente" es el espacio del aquí y ahora, es decir el de la producción musical: disco, gira y festival. «El Futuro» es inmediato, apunta a días concretos, se anticipa en meses y hablan de una nueva era de forma abstracta. «El Pasado» es la temporalidad de mayor peso, aunque no es homogéneo, se reparte entre la inmediatez del pasado próximo, las décadas anteriores y el regreso de cantantes y canciones.

\begin{tabular}{|c|c|c|c|c|c|c|c|c|}
\hline & \multicolumn{3}{|c|}{ Pasado } & El pasado en el presente & Presente & \multicolumn{3}{|c|}{ Futuro } \\
\hline Número total & \multicolumn{3}{|c|}{16} & 5 & & \multicolumn{3}{|c|}{5} \\
\hline Periodo & meses & años & otros & regreso & & día & mes & era \\
\hline Número & 5 & 5 & 1 & 5 & 4 & 1 & 3 & 1 \\
\hline
\end{tabular}

Tabla 3: Pasado, presente y futuro en cifras

Contexto espacial ¿Dónde ocurre? Las referencias espaciales nos remiten a 8 países -Estados Unidos, Argentina, Colombia, Bolivia, Reino Unido, Japón, Irak y España- y 8 ciudades, las españolas son todas de la mitad norte -Zaragoza, Barcelona, Madrid, San Sebastián y Santiago.

Contexto mediático. ¿Por medio de qué soporte? Los medios de comunicación y ficción destacados son el cine, otras revistas, la radio y, el más nombrado, la televisión.

\section{CONCLUSIONES}

Como contestación a nuestra pregunta de investigación sobre qué es música y con qué se relaciona para las dos revistas musicales de mayor tirada en España, hemos tomado como corriente de pensamiento el interaccionismo simbólico y como referencia las preguntas de Taylor: ¿Cómo se definen las personas a sí mismas y a otros, a sus escenarios y sus actividades? ¿Cómo se desarrollan y cambian las definiciones y perspectivas de la gente? ¿Cuál es el ajuste entre las diferentes perspectivas de distintas personas? ¿Cuál es el ajuste entre las perspectivas de la gente y sus actividades? ¿Cómo tratan las personas con las discrepancias entre sus perspectivas y actividades?

Por medio de un proceso inductivo basado en la significatividad y el vaciado progresivo: elección del entorno pop, elección de las revistas musicales de gran tirada y selección de editoriales de 2007 firmados por los directores de cada revista, hemos seleccionado los indicadores: identificación, comprensión, función social, difusión, finalidad y contexto. Como conclusiones generales podemos decir en primer lugar que las revistas musicales no tienen un público homogéneo, Rolling Stone sitúa una franja de edad, por gustos y poder adquisitivo, observable por indicadores de consumo, sensiblemente mayor a La Revista 40.

¿De qué hablan? Las revistas hablan especialmente de ellas mismas seguidas de cantantes y canciones. Los cantantes se identifican por atributos musicales y no musicales con referencias a rasgos físicos, de personalidad y de género. Las mujeres tienen una identificación por el físico sensiblemente mayor a los hombres. Los atributos musicales hablan del reconocimiento musical y social de los cantantes, así como de fidelidad al estilo y autenticidad. En cuanto a sus referencias más significativas, observadas por la forma en que la revista habla de lo que hacen los cantantes o provocan en otros, los resultados remiten al gusto, capacidad conmovedora de la música, sentido crítico y ser precursores. Por último, las canciones se identifican por su valoración musical (las mejores) y por su posición destacada en el disco al que pertenece (single)

¿Qué dicen? Las revistas musicales tienen una extensión media de 150 páginas pero hablan muy poco de música. Las pocas referencias que aparecen hablan de géneros del entorno del pop rock y escasas alusiones a elementos del lenguaje musical como el estribillo, aludiendo a la letra, el ataque 
como forma de reconocimiento melódico por las notas iniciales, y una sola referencia a la música como lenguaje con un sesgo innativista: "el don de la explosión creadora "no han tenido que aprender el lenguaje lo llevaban dentro" Los 40 (2007, 33:2). Sin embargo la creación de la música como proceso se desarrolla ampliamente en las revistas, este comienza con la idea musical y termina en el producto acabado. En este proceso, la grabación es el elemento dinámico, utilizado tanto por creadores como por productores que son los que finalmente materializan la música y la hacen permanente. La grabación es, por tanto, la piedra angular de la edición que permite, mediante la reordenación del material inicial, llegar al disco como producto acabado en un soporte comerciable.

¿Qué les preocupa? Entre sus páginas se pueden leer algunos textos sobre la política norteamericana, la mujer trabajadora, la ecología y el compromiso social, en que destacamos los comentarios políticamente incorrectos de los Simpson. En este espacio de las preocupaciones sociales hablan del valor mediador de la música como vehículo de poemas, metáforas, valores universales y muestran, también, de algunas relaciones ambiguas, como el caso de la descripción de un macro festival para sensibilizar sobre el cambio climático que después es convertido en número uno en ventas. Como identificador social del grupo de iguales, las revistas son fieles a su público potencial, es decir a sus clientes. La revista Rolling Stone, con la portada de John Lennon y Yoko Ono, se define a sí misma como emblema de toda una generación.

¿Dónde se muestra? La música se difunde en dos grandes espacios con igual número de referencias en ambos casos: el directo y los discos. El primero abarca desde las actuaciones y conciertos de carácter único, festivales o, como en el caso de las giras, repeticiones del mismo concierto en territorios más o menos extensos durante periodos que pueden ir desde una semana hasta varios años. El disco, sin embargo, admite muchos menos matices, sólo se habla de uno, el último, el resto no tiene presencia y por tanto existencia. De nuevo, ambos aspectos -gira y último disco- se encuentran relacionados, puesto que un grupo o cantante únicamente inicia una gira cuando tiene el disco en el mercado o en sus puertas. En cuanto a cantidades y cifras, podemos decir que los millones corresponden a los receptores, la fama se mide en minutos y los músicos se cuentan por decenas.

¿Para qué lo hacen? Destacan dos finalidades, la venta y las listas. Las revistas someten a listas todos los elementos que se puedan asociar a canciones. Hay listas de éxitos, ventas, clásicos, descargas, listas nacionales, extranjeras, lista de novedades, listas para pertenecer a las listas y un largo etc. Este patrón es una de las bases de las radio-fórmulas cuyo origen fue la MTV norteamericana. Fue importada a España y adoptada por Los 40 Principales que pasó, de ser un programa, a una radio-fórmula con emisión las 24 horas del día, al que han seguido otras como Cadena Dial o Cadena 100. Su vinculación a las ventas no es explícita, no hablan directamente de ellas, pero sí seleccionan los cantantes por los discos que están en venta, y realizan reportajes únicamente a aquellos que están en promoción. Las revistas, en líneas generales, toman como referencia el mundo del éxito y no se observa ninguna referencia al fracaso, únicamente se les permite, de nuevo, a los Simpson como espacio de ficción.

Los contextos: ¿Dónde ocurre? ¿A qué mundos remite? Hemos podido reconstruir cinco contextos por medio de los editoriales: el contexto espacial, el temporal, el mediático, el grupo de iguales y los mundos posibles. El contexto temporal habla de espacios de tiempo comprendidos entre el momento y la era, y una apuesta clara por el pasado del que hablan en 16 ocasiones frente a la 9 del presente y 5 del futuro. El pasado habla de meses y años, pero el periodo más nombrado es el de dos a tres décadas atrás, es decir, el periodo comprendido entre los ' 80 y los ' 90 . En cuanto al presente, aparece como dos espacios de acción diferentes, uno que habla de eventos: conciertos, discos, entrevistas, ensayos y series, y el segundo, con el mismo número de referencias, del regreso de cantantes y canciones. Por último, el futuro es considerado de forma inminente en términos de 
meses o bien como una aspiración: "una nueva era”. El ámbito geográfico del que hablan es mayoritariamente de habla inglesa y española referido este último a ocho ciudades de la mitad norte. Los espacios de divulgación y ficción son la radio, las revistas musicales, el cine y, de forma mayoritaria, la televisión.

Así, pues, la música en las revistas apuesta por grupos, cantantes consolidados y lanzamientos de discos, servidos en grandes formatos, carácter autorreferencial, y mucho peso publicitario que se definen, en ocasiones, como estilo de vida. En el ámbito cognitivo las revistas no ayudan a la comprensión de la música como fenómeno social vinculado a la especificidad de su propio lenguaje. Su significatividad como discurso portador de sentido tiene un alto componente emocional, proyectivo y de identificación, una escasa apuesta por la creación como forma de expresión, y un nivel crítico bajo.

\section{SUGERENCIAS PARA FUTURAS INVESTIGACIONES}

El estudio tuvo como intención saber de qué música hablan las revistas musicales y con que aspectos educativos las podemos relacionar, pero las revistas musicales no hablan de música. Las revistas analizadas ofrecen un mundo extremadamente pequeño, centrado en el triunfo y la inmediatez, algunos artistas y una gran industria a defender. El sujeto de la educación no encuentra su imagen en la revista. Quien sí la encuentra es el cliente que, por medio de su gran envoltorio, siempre asocia música con éxito, venta, luces, y en todo este conjunto, el lugar para la crítica sólo se contempla en la ficción, representada por los Simpson. El espacio de la música en las revistas es el del héroe como producto acabado surgido del propio sistema.

La música ocupa en nuestras escuelas e institutos un espacio de expresión, creación, producción y comprensión, y también cada vez menos páginas en los proyectos curriculares y la legislación educativa. Pero, a su vez, mueve una gran industria y espacios de opinión con los que se identifican millones de niños y jóvenes. Nosotros, en este artículo, hemos tomado uno de ellos, el espacio musical, social y cultural que generan, como la punta de iceberg del aparato mediático que sintetizan en sus páginas las grandes industrias del ocio y el espectáculo: las revistas musicales. En estas líneas hemos dado cuenta de dos de ellas, Rolling Stone y La Revista 40, como un inicio investigador del que hemos realizado un primer análisis y para cuya profundización proponemos tres líneas futuras de acción. En primer lugar esperamos que este pequeño mapa dé un poco de visibilidad sobre el imaginario social de la música y sus repercusiones para la educación. En segundo lugar, desde el ámbito estrictamente educativo, si las revistas sustituyen al mundo y por este mismo mecanismo lo generan como modos de representación (ECO, 1967; GOMBRICH, 1979), todos los agentes educativos están implicados en sus consecuencias. Por ello, esperamos que este estudio pueda contribuir como punto de atención sobre la responsabilidad de aquellos que determinan las grandes líneas del currículum obligatorio de la escolaridad. La sociedad actual necesita respuestas sólidas y fundamentadas para dar voz, formas de expresión, comprensión y capacidad crítica al ciudadano, siendo la música una manifestación cultural con uno de los imaginarios aglutinador de valores y tendencias más destacados. Finalmente, proponemos para futuras investigaciones, una tercera vía sobre la práctica educativa. Las revistas también hablan a la educación musical interpelando, además de a la legislación y a las políticas educativas, al profesorado, mostrando la cara más real de lo que la música es, o representa hoy en día para el sector más joven. Lo hacen con una voz fuerte, colores llamativos, modelos de héroe prefabricados, escasa variedad de géneros y estilos musicales y poca música, así como un elaborado proceso de edición y difusión. Si una de las finalidades más incuestionables de la educación es la de crear canales de comprensión de la realidad, nos gustaría, tomando los resultados del estudio como una 
primera referencia, iniciar la búsqueda de alternativas que ayuden a que la educación tenga entre sus funciones a través de la música y parafraseando a FREIRE (1980), la expresión musical como práctica de la libertad.

\section{Referencias}

Álvarez, J. L. (2003): Cómo hacer investigación cualitativa. México, Paidós.

BÁEZ, J. (2007): Investigación cualitativa. Madrid, Esic.

BARTHES, R. (1980): Mitologías. Madrid, Siglo XXI.

Blumer, H. (1969): Interaccionismo simbólico: Perspectiva y método. Barcelona, Hora.

Comunicar, 23. Consultado en www.revistacomunicar.com.

MINISTERIO DE EDUCACIÓN Y CIENCIA (2006): Real Decreto 1513/2006, de 7 de diciembre, por el que se establecen las enseñanzas mínimas de la Educación Primaria. Madrid, Boletín Oficial del Estado.

MINISTERIO DE EDUCACIÓN Y CIENCIA (2007): Real Decreto 1631/2006, de 29 de diciembre, por el que se establecen las enseñanzas mínimas correspondientes a la Educación Secundaria Obligatoria. Madrid, Boletín Oficial del Estado.

CASTORIADIS, C. (1989): La institución imaginaria de la sociedad. Barcelona, Tusquets.

ChARMAZ, K. (2000): Grounded theory: Objectivist and constructivist methods. Thousand Oaks (California), Sage.

CROTTY, M. (1998). The foundations of social research: Meaning and perspective in the research process. Londres, Sage.

CRUCES, F. (2004): "Música y ciudad: Definiciones, procesos y prospectivas". Revista Transcultural de Música, 8. Consultado en http://www.sibetrans.com/trans/trans8/cruces.htm.

Del Río, P.; DEL Río, M. (2008): “La construcción de la realidad por la infancia a través de su dieta televisiva". Comunicar, 31, 99-108.

DeWEY, J. (2008): El arte como experiencia, Barcelona, Paidós.

ECO, U. (1967): La estrategia de la ilusión. Barcelona, Lumen.

Eufonía. Didáctica de la Música, 12.

FRUTOS EsteBAN, F. J. (2008): "El análisis de contenido y la organización de repertorios culturales: El caso de las placas de linterna mágica". Revista Latina de Comunicación Social, 63, $265-$ 276. Consultado en http://www.ull.es/publicaciones/latina/ 2008/21 30 Salamanca/ Francisco Javier Frutos.html.

FLICK, U. (2007): Introducción a la investigación cualitativa. Madrid, Morata.

FREIRE, P. (1980): La educación como práctica de la libertad. México, Siglo XXI.

GARFINKEL, H. (1967): Studies in ethnomethodology. New Jersey, Prentice Hall.

Gimeno SACRistán, J.; PÉrez GómeZ, A. (1983): La enseñanza: su teoría y su práctica. Madrid, Akal.

Glaser, B. G.; STRAUSS, A. L. (1967): The discovery of grounded theory. Nueva York, Aldine.

GOMBRICH, E. H. (1979): Arte e ilusión. Barcelona, Gustavo Gili.

Goodman, N (1978): Maneras de hacer mundos. Madrid, La Balsa de la Medusa.

HeNNION, A. (2010): "Gustos musicales: De una sociología de la mediación a una pragmática del gusto". Comunicar, 34, 25-33.

JAMES, W. (1961): El significado de la verdad. Madrid, Aguilar. 
Kelle, U. (1995): Computer-aided qualitative data analysis. Londres, Sage.

KrIPPENDORFF, K, (1990): Metodología de análisis de contenido. Teoría y práctica. Barcelona, Paidós.

LACAN, J. (2009): Escritos (Vol. 2). México, Siglo XXI.

La Revista 40 (2007), 28, 29, 30, 31, 32.

LÉVI-STRAUSS, C. (1995): Antropología estructural. Barcelona, Paidós.

Litle, P.; ZuCKerman, M. (1986): “Sensation seeking and music preferences”. Personality and Individual Differences, 7(4), 575-578.

Muñoz, J. (2003). Análisis cualitativo de datos textuales con ATLAS/ti. Barcelona: Universidad Autónoma de Barcelona. Consultado el 28/01/2009 en www.ugr.es/ textinfor/documentos /manualatlas.pdf.

Patton, M. Q. (2002): Qualitative research \& evaluation methods. Thousand Oaks (California), Sage.

PeIRCE, C. (1988): Teoría de los signos. Barcelona, Paidós.

PELINSKY, R.(2007): "El oído alerta: Modos de escuchar el entorno sonoro". En: Actas del I Encuentro Iberoamericano sobre Paisajes Sonoros. Consultado el 25/9/2009 en http://cvc.cervantes.es/artes/paisajes sonoros/p sonoros01/pelinski/pelinski 01.htm.

Pérez, J. E.; Escudero, J. (2004). Análisis de la realidad local: Técnicas y métodos de investigación desde la animación sociocultural. Madrid, Narcea.

Porta, A. (2007): Músicas públicas, escuchas privadas. Hacia una lectura de la música popular contemporánea. Barcelona, Servei de Publicacions de la Universitat Autònoma de Barcelona.

PORTA, A. [ed.] (2010): ¿Qué escuchan los niños en la TV? Resultados del estudio realizado sobre la escucha de la programación infantil en una muestra de la cultura hispánica. Castellón. UJI/Rivera

PORTA, A. (2011): "La oferta musical de la programación infantil de TVE como universo audible. Un estudio educativo de su banda sonora". Comunicar, 37, 177-185.

Rolling Stone, 90, 91, 92, 93, 94.

SANDIN, P. (2003): Investigación cualitativa en educación. Madrid, McGrawHill.

SAussure, F. (1945): Curso de lingüística general. Buenos Aires, Losada.

SCHUTZ, A. (1962): El problema de la realidad social. Buenos Aires, Amorrortu.

SCHÜTZ, A. (1967): The phenomenology of the social world. Evanston (Illinois), North-Western University Press.

STRAuss, A.; CORBIN, J. (1998): Basics of qualitative research: Techniques and procedures for developing grounded theory ( $2^{\mathrm{a}} \mathrm{ed}$.). Londres, Sage.

TANNER, J. (2008): "Our favourite melodies: Musical consumption and teenage lifestyles". The British Journal of Sociology, 59(1), 117-144.

TAYLOR, S. J.: BOGDAN R. (1992): Introducción a los métodos cualitativos de investigación. Barcelona, Paidós.

VygotsKi, L. (1981): The concept of activity in Soviet psychology. Nueva York, Sharpe. 